E. C. Reisinger ${ }^{1}$

M. Lademann ${ }^{1}$

R. Krause ${ }^{2}$

\title{
Antibiotika-assoziierte Diarrhoe
}

\section{Antibiotic-associated diarrhea}

Die Antibiotika-assoziierte Diarrhoe (AAD) ist eine häufige Nebenwirkung einer Antibiotika-Therapie. Sie ist durch mehr als drei weiche oder flüssige Stühle pro Tag definiert und kann sich wenige Stunden bis 2 Monate nach Antibiotika-Einnahme entwickeln. Die Häufigkeit variiert abhängig vom Antibiotikum und der Art der Applikation. Im Krankenhaus tritt eine Diarrhoe bei 2 - 7\% der mit Antibiotika behandelten Patienten auf (12).

\section{Infelktiöse Ursachen der AAD}

\section{Clostridium difficile}

Nur $10-20 \%$ der AAD werden durch Infektionen, insbesondere mit Clostridium (C.) difficile, hervorgerufen. Die normale Darmflora unterdrückt das Wachstum von C. difficile. Durch Antibiotika (meist Cephalosporine, Clindamycin und BreitspektrumPenicilline, seltener auch andere, inkl. Vancomycin und Metronidazol) wird die Darmflora verändert, wodurch $C$. difficile überwuchern kann. Toxin A (Enterotoxin) und/oder Toxin B (Cytotoxin) von $C$. difficile führen zusammen mit Zytokinen zur Entzündung der Darmschleimhaut.

Das klinische Spektrum reicht von asymptomatischen Infektionen, Diarrhoe ohne Kolitis, nicht-pseudomembranöser Kolitis mit oder ohne Diarrhoe, pseudomembranöser Kolitis bis hin zur fulminanten Kolitis und Megakolon. Bei pseudomembranöser Kolitis wird $C$. difficile in nahezu allen Fällen nachgewiesen. Bei etwa 5\% gesunder Erwachsener sowie bei bis zu 65\% asymptomatischer Neugeborener findet sich $C$. difficile im Stuhl.

Patienten mit mehr als drei akuten medizinischen Problemen, einer mindestens 20-tägigen Antibiotika-Therapie, einem Alter über 70 Jahre, Begleiterkrankungen oder AAD-Rezidive zeigen ein erhöhtes Risiko einer symptomatischen Infektion mit $C$. difficile oder einen schwereren Verlauf (12).

\section{Clostridium perfringens}

C. perfringens kann eine AAD, oft bei älteren Patienten, verursachen. Meist werden bei AAD andere Serotypen als bei Lebensmittelvergiftungen nachgewiesen. In einer kürzlich erschienenen Arbeit wurde $C$. perfringens in $8 \%$, C. difficile in $16 \%$ und beide Ereger in $2 \%$ im Stuhl bei AAD gefunden (1).

\section{Akute segmental-hämorrhagische Penicillin-assoziierte Kolitis}

Nach oraler Therapie mit Penicillin oder auch mit Diclofenac kann eine akute hämorrhagische Diarrhoe mit schmerzhaften abdominellen Krämpfen auftreten. Als Ursache findet sich eine Überwucherung von Klebsiella oxytoca.

\section{Candida spp.}

Nach Antibiotika-Therapie kann es zu Fehlbesiedlung mit hohen Keimzahlen von Candida sp. ( $\geq 10^{5} \mathrm{CFU} / \mathrm{ml}$ ) im Darm kommen. Neuere Daten mit großen Fallzahlen belegen, dass erhöhte Keimzahlen von Candida spp. durch die Antibiotikatherapie bzw. durch den Durchfall selbst begünstigt werden und nicht umgekehrt (4). Es wurde gezeigt, dass Candida in Stuhlflüssigkeit von AAD-Patienten besser wächst als in der Stuhlflüssigkeit von gesunden Patienten (6). Auch die wesentlichen Virulenzfaktoren von Candida, sezernierte Aspartyl-Proteinasen (Saps) und Phospholipasen, spielen keine Rolle bei der Entstehung der AAD (5). Ältere Studien, wonach eine Überwucherung mit Candida Durchfall verursachen soll, wurden damit widerlegt.

\section{Salmonella spp. und Staphylococcus aureus}

Ähnlich wie bei Klebsiella oxytoca können durch z.B. Penicillin bereits im Darm vorhandene resistente Salmonellen selektioniert werden und zu Durchfallepisoden führen. Früheren Berichten zufolge kann Staphylococcus aureus eine AAD verursachen, in neueren Studien wurde dies jedoch nicht bestätigt.

${ }^{1}$ Abteilung für Tropenmedizin und Infektionskrankheiten, Klinik für Innere Medizin, Universität Rostock

${ }^{2}$ Abteilung für Infektiologie, Medizinische Universitätsklinik, Medizinische Universität Graz 
kurzgefasst: Nur etwa 10-20\% aller AAD werden durch $C$. difficile hervorgerufen. Bei gesunden Erwachsenen findet sich $C$. difficile bei $5 \%$, bei pseudomembranöser Kolitis in nahezu $\mathbf{1 0 0 \%}$ im Stuhl. Ein Fall von durch $C$. perfringens verursachter AAD kommt auf zwei Fälle von $C$. difficile. Durch Penicillin oder Amoxicillin können bereits im Darm vorhandene resistente Klebsiella oxytoca oder Salmonella spp. selektioniert werden. Candida spp. und Staph. aureus verursachen keine AAD.

\section{Störungen der Darmflora}

\section{Kohlehydrat-Metabolismus}

Die normale Darmflora metabolisiert nicht resorbierbare Kohlehydrate zu resorbierbaren kurzkettigen Fettsäuren. Bei durch Antibiotika gestörter Darmflora kumulieren die nicht resorbierbaren großmolekularen Kohlehydrate (Fruktose, Sorbitol) und führen zu osmotischer Diarrhoe (2).

\section{Verminderter Gallensäuremetabolismus}

Primäre Gallensäuren, die im Dünndarm nicht resorbiert wurden, werden von grampositiven anaeroben Stäbchen der Dickdarmflora zu sekundären Gallensäuren dehydroxyliert. Eine Reduktion dieser Bakterien durch Antibiotika bewirkt eine Zunahme der stark sekretorisch wirkenden primären Gallensäuren im Dickdarm und führt zu sekretorischer Diarrhoe (8).

\section{Beispiele für direkten Antibiotika-Effekt auf Motilität und Schleimhäute}

Erythromycin bindet an Motilin-Rezeptoren und bewirkt so Kontraktionen im Magen und Duodenum, damit beschleunigte Magenentleerung und funktionelle Diarrhoe. Auch für Clavulansäure wurde eine Motilitätssteigerung des Dünndarmes gezeigt.

7-11 Tage nach Gabe von Neomycin führen entzündliche Veränderungen an der intestinalen Schleimhaut zu gastrointestinalen Symptomen, Malabsorption und Steatorrhoe.

kurzgefasst: Die Antibiotika-assoziierte Diarrhoe kann durch eine Störung der Darmflora ausgelöst werden, die zu einer Kohlehydrat- und Gallensäurenmalabsorption führt. Weitere Ursachen sind eine pharmakologische Beeinflussung der Darmmotilität sowie allergisch-toxische Effekte von Antibiotika an der intestinalen Mucosa.

\section{Diagnose}

Zum Nachweis von $C$. difficile wird meist ein Schnelltest (EIA oder Latex-Test) mit einem spezifischen Test zum Nachweis des Erregers oder des Toxin B kombiniert. Der Stuhl muss sofort untersucht oder eisgekühlt werden. Durch die Kühlung kann der Toxin-Titer absinken. Die quantitative Bestimmung von Candida spp. im Stuhl ist obsolet (4).

Ist der Stuhl bei Patienten mit schwerer Symptomatik für $C$. difficile negativ, sollten $C$. perfringens, Klebsiella oxytoca und Salmonella spp. als seltene, aber mögliche Erreger in Betracht gezo- gen werden. Bei Patienten mit schwerer AAD und negativen Clostridien-Tests sollte eine Kolonoskopie angestrebt werden. Mittels Sigmoidoskopie alleine kann eine pseudomembranöse Kolitis oder segmental-hämorrhagische Kolitis nicht ausgeschlossen werden.

Störungen des Kohlehydrat- oder Gallensäuremetabolismus können durch Analyse der Kohlehydrate, kurzkettigen Fettsäuren oder Gallensäuren im Stuhl nachgewiesen werden. Aufgrund der meist selbstlimitierenden Natur dieser Diarrhoen ist diese aufwendige Diagnostik jedoch nicht notwendig.

kurzgefasst: $C$. difficile wird mit einem Schnelltest und einem spezifischen Test im Stuhl gesucht. Bei schwerer Symptomatik und negativen Clostridien-Tests sollte eine Kolonoskopie durchgeführt und $C$. perfringens, Klebsiella oxytoca und Salmonella sp. als seltene Erreger in Betracht gezogen werden.

\section{Prophylaxe}

Störungen des Kohlehydratmetabolismus können durch Weglassen von schlecht-resorbierbaren Kohlehydraten wie Fruktose und Sorbitol (in Früchten oder als Süßstoffe) oder NahrungsFasern (z.B. Pectine oder Guar in Karotten, Kohl, Bohnen u.a.) vermieden werden.

Saccharomyces boulardii (Perenterol ${ }^{\mathrm{R}}$ ) und Enterococcus SF68 $\left(\right.$ Bioflorin $^{\mathrm{R}}$ ) können das Auftreten von AAD bei verschiedenen Antibiotika-Therapien reduzieren (3).

Wenn möglich sollten Patienten mit C.-difficile-Nachweis zumindest für die Dauer der Diarrhoe in Einzelzimmer untergebracht werden. Das Tragen von Handschuhen und Händewaschen zwischen Kontakt mit verschiedenen Patienten ist obligatorisch.

Da die Antikörperbildung gegen C.-difficile-Toxin A während einer C.-difficile-Durchfallepisode vor einem Rezidiv schützen kann, wurde ein Toxoid-Impfstoff entwickelt, der in klinischen Studien bisher ermutigende Ergebnisse zeigt (10).

kurzgefasst: Die Aufnahme von kaum resorbierbaren Kohlehydraten (z.B. Sorbitol, Fruktose) und pflanzlichen Fasern (z.B. Pectine oder Guar) sollte vermieden werden. Probiotika können das Auftreten der AAD reduzieren.

\section{Therapie}

Wenn die AAD-auslösende antibiotische Therapie wegen einer zugrundeliegenden Infektion weitergeführt werden muss, dann sollte auf ein Antibiotikum gewechselt werden, das mit geringerem AAD-Risiko behaftet ist. Dazu zählen Quinolone, Cotrimoxazol, Tetrazykline, Aminoglykoside, Metronidazol, Vancomycin, Linezolid, Sulfonamide u.a. Schwere Fälle von C. difficile-positiven Diarrhoen müssen antibiotisch therapiert werden. Metronidazol, Vancomycin, Teicoplanin, Fusidinsäure und Bacitra- 
Tab.1 Antibiotische Therapie schwerer C. difficile-assoziierter Diarrhoe.

\begin{tabular}{lll}
\hline Antibiotikum & Dosierung & Rezidivrate \\
\hline Metronidazol & $3 \times 500 \mathrm{mg}$ für 7-14 Tage & $16 \%$ \\
Vancomycin & $4 \times 125 \mathrm{mg}$ für 7-14 Tage & $16 \%$ \\
Teicoplanin & $200 \mathrm{mg} /$ Tag für 7-14 Tage & $7 \%$ \\
Fusidinsäure & $1 \times 500 \mathrm{mg}$ für 7-14 Tage & $28 \%$ \\
Bacitracin & $4 \times 25000$ Units für 7-14 Tage &
\end{tabular}

cin zeigen einen ähnlichen therapeutischen Nutzen (Tab.1). Antibiotikum der ersten Wahl ist Metronidazol. Durch Vancomycin, das bei gleicher Rezidivrate (16\%) wesentlich teurer als Metronidazol ist, besteht die Gefahr der Resistenzentwicklung insbesondere bei Enterokokken. Teicoplanin, das teuerste aller bei AAD eingesetzten Antibiotika, hat mit 7\% die geringste Rezidivrate. Rezidive können mit dem gleichen oder einem anderen Antibiotikum behandelt werden (11). Von 415 spanischen C.-difficile-Isolaten waren 6,3\% resistent gegen Metronidazol und 3,1\% intermediär resistent gegen Vancomycin (9).

Saccharomyces boulardii kann C.-difficile-Toxin A und B proteolytisch verdauen. Die Gabe von Saccharomyces boulardii zusammen mit $C$. difficile wirksamen Antibiotika kann die Zahl der C.difficile-Rezidive vermindern (7). Entsprechend den Ergebnissen kleinerer Studien kann durch Gabe von Bifidobacterium longum die Zahl der Erythromycin-induzierte Diarrhoen und durch Lactobacillus die Zahl von Ampicillin-induzierten Diarrhoen vermindert werden (3).

\section{kurzgefasst: Wenn die antibiotische Therapie weiterge- führt werden muss, dann sollte auf ein Antibiotikum mit geringerem AAD-Risiko gewechselt werden.}

Metronidazol ist Therapie der ersten Wahl bei schwerer durch $C$. difficile verursachter AAD. Rezidive können mit dem gleichen oder einem anderen Antibiotikum behandelt werden. Saccharomyces boulardii kann zusammen mit $C$. difficile wirksamen Antibiotika die Zahl der $C$. difficile Rezidive vermindern.

Fazit

Die Antibiotika-assoziierte Diarrhoe (AAD) ist eine häufig auftretende Nebenwirkung einer Antibiotika-Therapie und kann sich wenige Stunden bis 2 Monate nach AntibiotikaEinnahme entwickeln. Sie ist klinisch relevant, wenn mehr als drei weiche oder flüssige Stühle pro Tag abgesetzt werden. Die Häufigkeit variiert - abhängig vom Antibiotikum und der Art der Applikation - und liegt bei Krankenhauspatienten zwischen $2 \%$ und $7 \%$.

- Verschiedene Mechanismen wie Schädigung der normalen Darmflora und damit verbundene Störungen des Kohlehydrat- und Gallensäuremetabolismus, allergisch-toxische Effekte an der intestinalen Mucosa, pharmakologische Beeinflussung der Darmmotilität und Infektionen durch Über- wucherung von pathogenen Mikroorganismen können Ursache der AAD sein.

- Stuhluntersuchungen, insbesondere der Nachweis von $C$. difficile, C. perfringens, Klebsiella oxytoca und Salmonellen tragen zur Diagnosefindung bei.

- Metronidazol ist Mittel der ersten Wahl zur Therapie der schweren durch C.difficile verursachten AAD. Probiotika können das Auftreten von AAD reduzieren.

- Rationaler Einsatz von Antibiotika ist die wirksamste Maßnahme zur Vermeidung der AAD.

\section{Literatur}

${ }^{1}$ Asha NJ, Wilcox MH. Laboratory diagnosis of Clostridium perfringens antibiotic associated diarrhoea. J Med Microbiol 2002; 51: 891-894

${ }^{2}$ Hammer HF, Fine KD, Santa Ana CA, Porter JL, Schiller LR, Fordtran JS. Carbohydrate malabsorption: its measurement and its contribution to diarrhea. J Clin Invest 1990; 86: 1936-1944

${ }^{3}$ Högenauer C, Hammer HF, Krejs GJ, Reisinger EC. Mechanisms and management of antibiotic associated diarrhea. Clin Infect Dis 1998; 27: 702-710

${ }^{4}$ Krause R, Schwab E, Bachhiesl D, Wenisch C, Krejs GJ, Reisinger EC. Role of Candida in Antibiotic-Associated Diarrhea. J Inf Dis 2001; 184: 1065-1069

${ }^{5}$ Krause R, Haberl R, Strempfl C et al. Intestinal Candida phospholipase is not elevated in patients with antibiotic-associated diarrhea. Scand J Infect Dis 2002; 34: 815-816

${ }^{6}$ Krause R, Krejs GJ, Wenisch C, Reisinger EC. Elevated fecal Candida counts in patients with antibiotic-associated diarrhea: the role of soluble fecal substances. Clin Lab Diagn Immunol 2003; 10: 167-168

7 Mc Farland LV, Surawicz CM, Greenberg RN et al. A randomized placebo-controlled trial of Saccharomyces boulardii in combination with standard antibiotics for Clostridium difficile disease. JAMA 1994; 271: 1913-1918

${ }^{8}$ Mc Junkin B, Fromm H, Sarva RP, Amin P. Factors in the mechanism of diarrhea in bile acid malabsorption: fecal $\mathrm{pH}$ - a key determinant. Gastroenterology 1981; 80: 1454-1464

9 Pelaez T, Alcala L, Alonso R, Rodriguez-Creixems M, Garcia Lechuz JM, Bouza E. Reassessment of Clostridium difficile susceptibility to metronidazole and vancomycin. Antimicrob Agents Chemother 2002; 46: 1647-1650

${ }^{10}$ Stoddart B, Wilcox MH. Clostridium difficile. Curr Opin Infect Dis 2002; 15: 513-518

11 Wenisch C, Parschalk B, Hasenhundl M, Hirschl AM, Graninger W. Comparison of vancomycin, teicoplanin, metronidazole, and fusidic acid for the treatment of Clostridium difficile-associated diarrhea. Clin Infect Dis 1996; 22: 813-818

12 Wistrom J, Norrby SR, Myhre EB et al. Frequency of antibiotic associated diarrhoea in 2462 antibiotic-treated hospitalized patients: a prospective study. J Antimicrob Chemother 2001; 47: 43-50 